\title{
Metacercarias del tipo Paramonilicaecum (Digenea: Didymozoidae), parásitos accidentales de elasmobranquios (Elasmobranchii) del golfo de México e identificación de metacercarias (Didymozoidae) de la Colección Nacional de Helmintos
}

\section{Metacercariae of the type Paramonilicaecum (Digenea: Didymozoidae), accidental parasites in elasmobranchs (Elasmobranchii) from the Mexican Gulf of Mexico and identification of metacercarias (Didymozoidae) of the Colección Nacional de Helmintos}

\author{
Erick Rodríguez-Ibarra, Scott Monks* y Griselda Pulido-Flores \\ Laboratorio de Morfología Animal, Centro de Investigaciones Biológicas, Universidad Autónoma del Estado de Hidalgo. Apartado postal 1-69, 42001 \\ Pachuca, Hidalgo, México. \\ *Correspondencia: smonks@uaeh.edu.mx
}

\begin{abstract}
Resumen. Como parte de un estudio sobre los helmintos parásitos de rayas (Elasmobranchii), se colectaron 12 ejemplares en 3 localidades del estado de Veracruz, México: Laguna de Tamiahua, Laguna de Tampamachoco y un arrecife de la costa del golfo de México. Se encontraron metacercarias identificadas como parásitos accidentales del tipo Paramonilicaecum (Digenea: Didymozoidae) en la válvula espiral de Dasyatis say (raya látigo chata), Gymnura micrura (raya cola de rata) y Rhinobatus lentiginosus (guitarra diablito). De manera complementaria, se actualizó la identificación de material perteneciente a Didymozoidae depositado en la Colección Nacional de Helmintos en el Instituto de Biología, Universidad Nacional Autónoma de México. Éste es el primer registro para México de metacercarias del tipo Paramonilicaecum en rayas.
\end{abstract}

Palabras clave: Didymozoidae, metacercaria, Elasmobranchii, Veracruz, Jalisco, México, Costa-Rica.

\begin{abstract}
During an ongoing study of the helminths of stingrays (Elasmobranchii), 12 stingrays were collected from 3 localities in the state of Veracruz, Mexico: Laguna de Tamiahua, Laguna de Tampamachoco, and a costal reef in the Gulf of Mexico. Metacercarias were collected from the spiral valves of Dasyatis say (bluntnose stingray), Gymnura micrura (lesser butterfly ray), and Rhinobatus lentiginosus (Atlantic guitarfish). The metacercarias, accidental parasites, were assigned to type Paramonilicaecum (Digenea: Didymozoidae). Material (Didymozoidae) previously deposited in the Colección Nacional de Helmintos, Instituto de Biología, Universidad Nacional Autónoma de México, was re-identified. This is the first report of a species in larval phase of the "Type" Paramonilicaecum from the Gulf of Mexico, Veracruz, and the first report from a Mexican stingray.
\end{abstract}

Key words: Didymozoidae, metacercaria, Elasmobranchii, Veracruz, Jalisco, Mexico, Costa-Rica.

Las formas larvarias de la familia Didymozoidae se encuentran ocasionalmente en el intestino de peces que actúan como terceros hospederos intermedios o paraténicos; también se han encontrado en calamares, quetognatos, copépodos y poliquetos (Gómez del Prado-Rosas et al., 2007; Pozdnyakov y Gibson, 2008; Køie y Lester, 1985; Madhavi, 1968). Recientemente, Pozdnyakov y Gibson (2008) establecieron un sistema de clasificación de acuerdo con los grupos nombrados "tipos" (que no deben con-

Recibido: 22 febrero 2010; aceptado: 10 agosto 2010 fundirse con nombres genéricos), basados en estructuras particulares. El objetivo del presente trabajo es registrar la presencia de metacercarias de una especie de didimozoide en los intestinos de algunas rayas de México y actualizar, de acuerdo con Pozdnyakov y Gibson (2008), la identificación de las metacercarias depositadas en la Colección Nacional de Helmintos (CNHE) en el Instituto de Biología, Universidad Nacional Autónoma de México (CNHE).

Como parte del estudio de helmintos parásitos de elasmobranquios de México se recolectaron 12 rayas a través de la captura comercial en 3 localidades del norte 
del estado de Veracruz, México, cerca de la boca del río Pantepec (laguna de Tamiahua, laguna de Tampamachoco y en un arrecife en la costa del golfo de México, $21^{\circ} 04^{\prime} 24.61^{\prime \prime} \mathrm{N}$ y $97^{\circ} 15^{\prime} 55.07^{\prime} \mathrm{O}$ ). Las rayas colectadas pertenecen a 4 familias; 3 son individuos de Dasyatis say (Lesueur, 1817) (raya látigo chata); 2 de D. sabina (Lesueur, 1824) (raya látigo de espina) (Dasyatidae); 2 de Gymnura micrura (Bloch y Schneider, 1801) (Gymnuridae: raya cola de rata); 1 de Dipturus oregoni (Bigelow y Schroeder, 1958) (Rajidae: raya diabla) y 4 de Rhinobatus lentiginosus (Garman, 1880) (Rhinobatidae: guitarra o diablito). Las metacercarias recolectadas fueron procesadas mediante las técnicas convencionales de acuerdo con Pritchard y Kruse (1982); los ejemplares de referencia se depositaron en 3 colecciones: la CNHE, la Colección de Helmintos (CHE) del Centro de Investigaciones Biológicas, Universidad Autónoma del Estado de Hidalgo y el Harold W. Manter Laboratory of Parasitology (HWML), University of Nebraska-Lincoln. Adicionalmente, se revisó material depositado en la CNHE por León-Règagnon et al. (1997; bahía de Chamela, Jalisco, 19³ 30' N y $105^{\circ} 06^{\prime}$ O, CNHE 2776-2778, 2780-2788) y por PérezPonce de León et al. (1998; Playa Hermosa, bahía Culebra, Costa Rica, $10^{\circ} 38^{\prime} \mathrm{N}$ y $85^{\circ} 38^{\prime} \mathrm{O}$, CNHE 3182 ), así como las figuras publicadas por Gómez del Prado-Rosas et al. (1999 y 2007).

Los 5 didimozoides recolectados en Veracruz (nuevo registro geográfico) se asignaron al tipo Paramonilicaecum de Kurochkin y Nikolaeva, 1978 (Fig. 1A; CNHE-72827284; HWML-49246; CHE-P00060) por la presencia de ventosa oral, más pequeña que el acetábulo, faringe pequeña, "Drüsenmagen" (una cámara localizada sobre la bifurcación del ciego) presente sin glándulas celulares rodeando la parte anterior del sistema alimentario, esófago largo y delgado, y ciegos formados por cámaras bien desarrolladas (Pozdnyakov y Gibson 2008). Las metacercarias presentan variación morfológica sólo en largo total y ancho, a pesar de parasitar 3 especies de hospederos (R. lentiginosus, G. micrura y D. say), posiblemente como resultado de su desarrollo distinto.

El estudio de los ejemplares registrados por LeónRègagnon et al. (1997) en Jalisco, México (Fig. 1B; CNHE: 2776-2778, 2780-2783, 2785, 2787 y 2788), y por Pérez-Ponce de León et al. (1998) (Fig. 1C; CNHE 3182) en Costa-Rica, permitió asignarlos al tipo Neotorticaecum por la presencia de ventosa oral, faringe y ausencia de "Drüsenmagen", bifurcación intestinal anterior al acetábulo, esófago corto y muy delgado, glándulas celulares rodeando la parte distal del esófago y la parte anterior del ciego, las cámaras del ciego se incrementan posteriormente en tamaño (Pozdnyakov y Gibson, 2008). Sin embargo, los ejemplares de Jalisco presentan la faringe rodeada de célu- las, mientras que los de Costa Rica carecen de ellas. Esta característica sugiere la posibilidad de que representen especies diferentes. Otro ejemplar de Jalisco se asignó al tipo Allomonilicaecum (Fig. 1D; CNHE: 2786) por la presencia de ventosa oral terminal, faringe, "Drüsenmagen" bien desarrollado, rodeado de células glándulares al igual que la parte anterior del ciego, esófago largo y delgado, ciegos curvos y desarrollo escaso de las cámaras (Pozdnyakov y Gibson, 2008). De acuerdo con Pozdnyakov y Gibson (2008) en este tipo larvario la ventosa oral es más grande que el acetábulo, rasgo que se presentó invertido en el ejemplar estudiado. No obstante, la consistencia del resto de sus características con las descritas para el tipo Allomonilicaecum y el escaso conocimiento actual de estas especies impide el establecimiento de otro tipo de larva con base en este único rasgo. Finalmente, no se realizó la asignación del ejemplar CNHE-2784, procedente de Jalisco, México, debido a su mal estado de conservación.

En México se han registrado algunos didimozoides para el mar Caribe; Torticaecum sp. [sic] en Serratosagitta serratodentata (Krohn, 1853) (Gómez del Prado-Rosas et al., 1999) y el tipo Monilicaecum [sic] en Flaccisagitta enflata (Grassi 1881) y S. serratodentata (Gómez del Prado-Rosas et al., 2007). Con base en su descripción y en la figura 1 del documento, los didimozoides registrados como Torticaecum sp. acreditan pertenecer al tipo Torticaecum por la presencia de faringe, ausencia de "Drüsenmagen”, bifurcación del ciego anterior al acetábulo y esófago delgado sin células glandulares. Sin embargo, de acuerdo con Pozdnyakov y Gibson (2008), no es posible asignarlos al género Torticaecum sin más información. Por el contrario, la identificación de los ejemplares registrados por Gómez del Prado-Rosas et al. (2007) como tipo Monilicaecum es problemática, ya que su morfología no coincide totalmente con la diagnosis propuesta por Pozdnyakov y Gibson (2008). De acuerdo con estos autores, el tipo Monilicaecum se caracteriza por la ausencia de faringe y presencia de un "Drüsenmagen" sin glándulas celulares, pero Gómez del Prado-Rosas et al. (2007) registraron una "faringe inconspicua, observada sólo en 4 ejemplares". De la misma forma, en la figura 1 de este trabajo, las metacercarias se esquematizan sin faringe y con "Drüsenmagen". Los ejemplares descritos con faringe y con "Drüsenmagen”, posiblemente pertenecen al tipo Paramonilicaecum. Sin embargo, los ejemplares de Gómez del Prado-Rosas et al. (1999; CNHE-3194) y los de Gómez del Prado-Rosas et al. (2007) no fueron depositados en la CNHE, imposibilitando su evaluación.

En este trabajo se establece el primer registro de didimozoides tipo Paramonilicaecum en México, como parásitos ocasionales de la válvula espiral de elasmobranquios. El ciclo de vida de los didimozoides es complejo, 


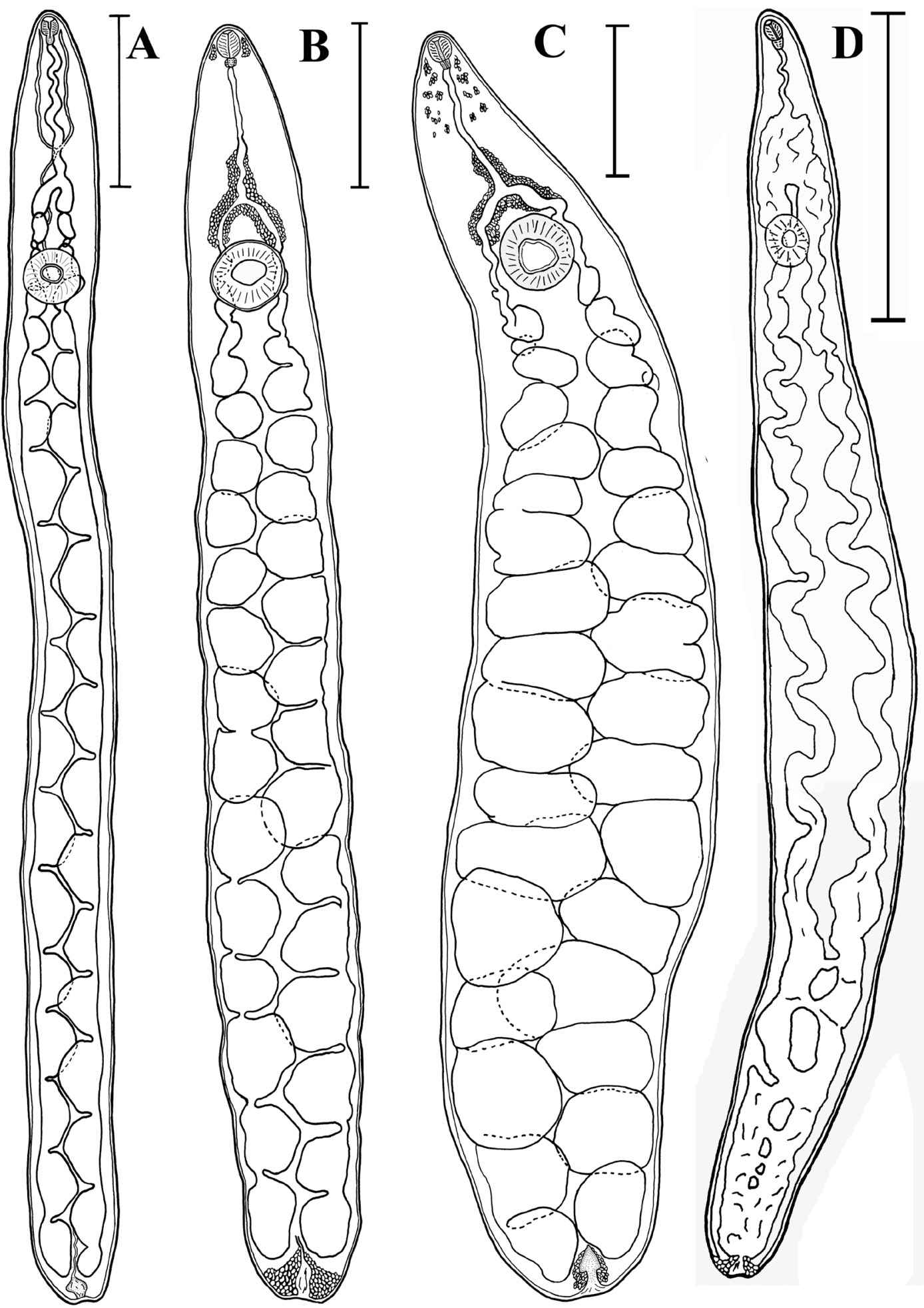

Figura 1. Morfología interna de larvas de didimozoides (vista ventral): A, tipo Paramonilicaecum (CNHE-7282)Veracruz; B, tipo Neotorticaecum (CNHE-2776), bahía de Chamela, Jalisco, México; C, tipo Neotorticaecum (CNHE-3182D), Playa Hermosa, bahía Culebra, Costa Rica; D, tipo Allomonicaecum (CNHE-2786), bahía de Chamela, Jalisco, México. Escala: $500 \mu \mathrm{m}$. 
requiere 4 hospederos para completarlo (Hochberg y Holt, 1990; Køie y Lester, 1985). La dieta de algunos elasmobranquios incluye crustáceos, copépodos y pequeños peces, siendo posible que al depredar a éstos adquieran la infección. Hasta el momento, no se han registrado didimozoides adultos como parásitos de elasmobranquios. La biología molecular ha abierto un campo importante en la determinación de especies de Didymozoidae (Anderson y Barker, 1993; Anderson y Cribb, 1994; Anderson y Barker, 1998), convirtiéndose en un método que puede ser de gran utilidad paralos miembros de esta familia, pero aún falta obtener secuencias de didimozoides adultos de México para aplicar la técnica en la identificación de estas metacercarias.

Los autores agradecen a Luis García-Prieto, curador de la CNHE y a Jorge Falcón Ordaz por la disponibilidad de su tiempo. A Marisela López-Ortega y María Alejandra López-Jiménez, Universidad Veracruzana, por su apoyo en la obtención de las rayas y por el acceso al laboratorio y a Francisco Javier Alemán-Muñoz por el apoyo en la colecta de ejemplares. El primer autor agradece al CONACyT por la beca nacional (Núm. 225995) concedida para estudios de la Maestría en Ciencias en Biodiversidad y Conservación (PNPC), Universidad Autónoma del Estado de Hidalgo.

\section{Literatura citada}

Anderson, G. R. y S. C. Barker. 1993. Species differentiation in the Didymozoidae (Digenea): Restriction fragment length differences in internal transcribed spacer and 5.8S ribosomal DNA. International Journal for Parasitology 23:133-136.

Anderson, G. R. y S. C. Barker. 1998. Inference of phylogeny and taxonomy within the Didymozoidae (Digenea) from the second internal transcribed spacer (ITS2) of ribosomal DNA. Systematic Parasitology 41:87-94.

Anderson, G. R. y T. H. Cribb. 1994. Five new didymozoid trematodes (Platyhelminthes, Digenea) from Australian platycephalid fishes. Zoologica Scripta 23:83-93.

Gómez del Prado-Rosas, M. d. C., J. Alvarez-Cadena, L. Segura-Puertas y R. Lamothe-Argumedo.1999. First record of Torticaecum sp. (Trematoda: Didymozoidae) in the chaetognath Serratosagitta serratodentata (Krohn, 1853) from Caribbean waters. Journal of Plankton Research 21:1005-1008.

Gómez del Prado-Rosas, M. d. C., J. N. Álvarez-Cadena, L. Segura-Puertas y R. Lamothe-Argumedo. 2007. Didymozoid Monilicaecum type trematodes in chaetognaths from the Mexican Caribbean Sea. Revista Mexicana de Biodiversidad 78:483-487.

Hochberg, M. E. y R. D. Holt. 1990. The coexistance of competing parasites. I. The role of cross-species infection. American Naturalist 136:517-541.

Køie, M. y R. J. G. Lester. 1985. Larval didymozoids (Trematoda) in fishes from Moreton Bay, Australia. Proceedings of the Helminthological Society of Washington 52:196-203.

León-Règagnon, V., G. Pérez-Ponce de León y R. LamotheArgumedo. 1997. Hemiuriformes de peces marinos de la Bahía de Chamela, México, con la descripción de una nueva expecie del género Hysterolecitha (Digenea: Hemiuridae: Lecithasterinae). Anales del Instituto de Biología, Universidad Nacional Autónoma de México, Serie Zoología 68:1-34.

Madhavi, R. 1968. A didymozoid metacercaria from the copepod, Paracalanus aculeatus Giesbrecht, from Bay of Bengal. Journal of Parasitology 54:6-29.

Pérez-Ponce de León, G., V. León-Regagnon y S. Monks. 1998. Theletrum lamothei sp. nov. (Digenea: Hemiuridae), parasite of Echidna nocturna in Cuajiniquil, Guanacaste Province, and other platyhelminths from marine fishes from Costa Rica. Revista Biológica Trópica 46:159-168.

Pozdnyakov, S. E. y D. I. Gibson. 2008. Family Didymozoidae Monticelli, 1888. In Keys to the Trematoda, R. A. Bray et al., (eds.). CAB International y Natural History Museum, London, p. 631-734.

Pritchard, M. H. y G. O. W. Kruse. 1982. The collection and preservation of animal parasites. University of Nebraska Press, Lincoln, 141 p. 